\title{
Successive Pilot Contamination Elimination in Multiantenna Multicell Networks
}

\author{
Thang X. Vu, Member, IEEE, Trinh Anh Vu, and Tony Q. S. Quek, Senior Member, IEEE
}

\begin{abstract}
This paper addresses the problem of channel estimation in time-division duplex (TDD) multicell cellular systems, where the performance of such systems is usually bounded by a bottleneck due to pilot contamination. We propose two channel estimation schemes that completely remove pilot contamination. The exact closed-form expression for average mean square error (MSE) of the proposed estimators is derived. More importantly, our proposed estimators do not need to know the second-order statistics of either desired user channels or interfering user channels. Finally, simulated results confirm gains over existing channel estimation schemes.
\end{abstract}

Index Terms-Channel estimation, pilot contamination, MIMO systems.

\section{INTRODUCTION}

$\mathbf{M}$ ULTIPLE-INPUT multiple-output (MIMO) have been widely deployed as the key technology in future mobile networks where adjacent cells share the same frequency in order to meet the exponential demand for data rate. However, full reuse of frequency at neighboring cells can cause estimated channel state information (CSI) in one cell to be contaminated by pilot training in other cells since the orthogonal property is usually not maintained among pilot sequences across different cells. Pilot contamination constitutes a performance bottleneck that impairs the effectiveness of MIMO system in cellular networks [1], [2].

The effects of pilot contamination in multicell MIMO systems with large number of base station (BS) antennas has been analyzed in [3], in which it was showed that pilot contamination still persists in large-scale MIMO systems. The authors in [4] proposed a coordinated estimation method that can eliminate pilot contamination when the number of BS antennas reaches infinity by exploiting the second-order statistics of the user channels. In this work, a covariance-aware pilot assignment strategy was proposed to deliver identical pilot sequences to a group of selected users. Consider practical constraints in which the number of BS antennas is usually limited, the authors in [5] proposed a time-shifted structure to alleviate the effect of pilot contamination by assigning orthogonal pilot transmissions across different cells. Base on this structure, the

Manuscript received June 19, 2014; revised August 18, 2014; accepted September 20, 2014. Date of publication October 3, 2014; date of current version December 17, 2014. This work was supported in part by the SRG ISTD under Grant 2012037, the SUTD-MIT International Design Centre under Grant IDSF1200106OH, and the Vietnam National University, Hanoi, under Research Project A. The associate editor coordinating the review of this paper and approving it for publication was B. Shim.

T. X. Vu and T. Q. S. Quek are with the Singapore University of Technology and Design, Singapore 138682 (e-mail: xuanthang_vu@ @utd.edu.sg; tonyquek@sutd.edu.sg).

T. A. Vu is with the Electronics and Telecommunications Department, VNU University of Engineering and Technology, Hanoi, Vietnam (e-mail: vuta@ vnu. edu.vn).

Digital Object Identifier 10.1109/LWC.2014.2361518 authors in [6] proposed time staggered pilots scheme which can asymptotically remove pilot contamination whenever pilot intervals at different cells do not overlap. However, to ensure the orthogonality among pilot transmissions across different cells, it requires a central controller to manage the staggering of pilot training in all cells, which obviously becomes challenging with large number of users and cells. It is worthy to remind that most of the above schemes must know not only the secondorder statistics of desired user channels, but also the secondorder statistics of interfering channels from users of different cells. Recently, the authors in [7] proposed a channel estimation strategy which can eliminate pilot contamination without the knowledge of any side-information. This strategy consists of an amalgam of downlink and uplink training. The drawback is that energy consumption on uplink training might frequently exceed the peak transmit power, and thus causes the system unreliable.

In this paper, we propose a novel pilot contamination elimination scheme applied to TDD multicell systems. The proposed scheme consists of consecutive pilot transmission phases in which each BS stays idle at one phase and repeatedly transmits pilot sequence in other phases. Compared to [4], our scheme do not need to know the covariance matrices of both the desired and interfering user channels. Compared to [7], our scheme saves $3 K$ pilot symbols and does not suffer from the peak power problem, where $K$ is the length of the pilot sequence. Two estimators are proposed to completely remove pilot contamination. The exact closed-form expression for average mean square error (MSE) of both estimators are provided. The gains over existing channel estimation scheme are confirmed via simulated results.

The rest of this paper is organized as follows. Section II describes the system model and pilot contamination problem. Section III gives details about our proposed pilot contamination elimination scheme and its analysis. Section IV presents the numerical and simulated results. Finally, Section V concludes the paper.

\section{SYSTEM MODEL}

The network consists of $L$ time-synchronized cells with unit fraction of spectrum reuse [4]. In each cell, one BS, which is equipped with $Q$ transmit/receive antennas, serves $M$ single-antenna users. Block fading model is assumed where the channel fading coefficients remain constant within a block, but independently change across different blocks. In practice, the block length depends on the coherence time. For each block, the channel is estimated during a channel estimation period.

\section{A. Channel Estimation for the Uplink}

Uplink channel estimation is acquired via synchronous transmission of pilot symbols from all users in the network. Denote by $\mathbf{x}_{l}^{i}=\left[x_{l, 1}^{i}, \ldots, x_{l, K}^{i}\right]^{T} \in \mathbb{C}^{K \times 1}$ the pilot sequence that 
comprises of $K$ pilot symbols transmitted by the $i$-th user at the $l$-th cell, with $(.)^{T}$ denotes the transpose operator. Each pilot symbol has unity energy. We assume that the pilot sequences within a cell are independent, e.g., $\mathbf{x}_{l}^{i^{H}} \mathbf{x}_{l}^{j}=K$ only if $i=j$ and $\mathbf{x}_{l}^{i H} \mathbf{x}_{l}^{j}=0$ for $i \neq j$, with $(.)^{H}$ denotes the Hermitian transpose. The pilot sequences at different cells might be correlated because the orthogonal property might not hold among a large number of users in the whole network [3]. Let $\mathbf{h}_{l, l^{\prime}}^{i}=$ $\left\{h_{l, l^{\prime}}^{i}[1], \ldots, h_{l, l^{\prime}}^{i}[Q]\right\}$, with $h_{l, l^{\prime}}^{i}[q] \sim \mathcal{C N}\left(0, \sigma_{h_{l, l^{\prime}}}^{2}\right), 1 \leq q \leq$ $Q$ be the channel fading vector from the $i$-th user at the $l$-th cell to the BS at the $l^{\prime}$-th cell. In this model, path loss is captured in $\sigma_{h_{l, l^{\prime}}}^{2}$. The received signal at the BS of cell $l$ is given as:

$$
\mathbf{Y}_{l}=\mathbf{X}_{l} \mathbf{H}_{l, l}+\sum_{l^{\prime}=1, l^{\prime} \neq l}^{L} \mathbf{X}_{l^{\prime}} \mathbf{H}_{l^{\prime}, l}+\mathbf{Z}_{l}
$$

where $\mathbf{H}_{l^{\prime}, l}=\left[\mathbf{h}_{l^{\prime}, l}^{1}{ }^{T}, \ldots, \mathbf{h}_{l^{\prime}, l}^{M^{T}}\right]^{T}$ is the $M \times Q$ matrix, $\mathbf{X}_{l}=$ $\left[\mathbf{x}_{l}^{1}, \ldots, \mathbf{x}_{l}^{M}\right]$ is the $K \times M$ pilot signal matrix at the $l$-th cell, and $\mathbf{Z}_{l}$ is a $K \times Q$ noise matrix, whose elements are i.i.d. complex Gaussian random variable with zero mean and variance $\sigma^{2}$.

In (1), the first term stands for the desired pilot signal transmitted from the users at cell $l$-th, and the second term stands for the inter-cell interference of the other $L-1$ cells. The conventional least square (LS) channel estimate is given as follows [7]:

$$
\widehat{\mathbf{H}}_{L S}=\left(\mathbf{X}_{l}^{H} \mathbf{X}_{l}\right)^{-1} \mathbf{X}_{l}^{H} \mathbf{Y}_{l}
$$

\section{B. Pilot Contamination}

Due to the lack of orthogonality of the pilot signals across the cells [4], the inter-cell interference is not completely cancelled out in (2). This results in pilot contamination which significantly degrades the channel estimation accuracy. In particular, if the pilot signals are fully reused in all cells, e.g., $\mathbf{X}_{l}=\mathbf{X}$ for $\forall l$, with $\mathbf{X}^{H} \mathbf{X}=K \mathbf{I}_{M}$, where $\mathbf{I}_{M}$ denotes the $M \times M$ unity matrix. Then the LS estimation becomes:

$$
\widehat{\mathbf{H}}_{L S}=\mathbf{H}_{l, l}+\sum_{l^{\prime}=1, l^{\prime} \neq l}^{L} \mathbf{H}_{l^{\prime}, l}+\frac{1}{K} \mathbf{X}^{H} \mathbf{Z}_{l} .
$$

A severe estimation error can be made by the second term in (3), especially when the average channel gain of interfered channels is comparable with that of the desired channel. For stronger minimum mean square error (MMSE) estimator, pilot contamination is still a big obstacle, even when the number of antennas tends to infinity [7]. A recently coordinated channel estimator based on coordinated pilot assignment strategy can only eliminate pilot contamination if the number of antennas reaches infinity, but still results in dominant estimation errors in finite antennas regime [4].

\section{Proposed Pilot Contamination ELIMINATION SCHEME}

We propose a channel estimation scheme that completely eliminates pilot contamination and does not require any second- order statistics. The proposed scheme takes place in $L+1$ pilot transmission phases (phase) which are obtained by dividing the channel estimation period by $L+1$. In the first phase, named phase 0 , all users at all cells transmit their assigned pilot sequence to their corresponding $\mathrm{BS}$ at the same time. We note that our scheme does not require the transmissions at all cells to be perfectly synchronized, but only the transmissions within a cell. In the next $L$ phases, each cell consecutively stays silent while all other cells are repeatedly transmitting. More specifically, at the $l$-th phase, all users in the $l$-th cell stays silent, while the users in others $L-1$ cells are transmitting their pilot. Because the estimation period is short (several to dozens of symbols), the silence of users has negligible effect on system throughput. Although having $L+1$ phases, each cell only transmits in $L$ phases. As a result, the proposed scheme consumes $L K$ pilot symbols. Compared with classical scheme [4], where the channel estimation period only consists one pilot transmission phase, the training phase (for one cell) in our scheme is $L+1$ times shorter. In other words, our scheme sacrifices pilot's length to obtain orthogonal pilot transmission among $L$ cells, resulting in smaller number of supportable users.

Without loss of generality, we assume that cell 1 is the cell of interest and focus on the processing at this cell. Let $\mathbf{H}_{1,1} \in \mathbb{C}^{M \times Q}$ and $\mathbf{H}_{l, 1} \in \mathbb{C}^{M \times Q}, 2 \leq l \leq L$ be the desired channel fading coefficients and the interfering channel fading coefficients (between $l$-th cell's users and BS 1), respectively. We assume the pilot signal $\mathbf{X}$ is fully reused by every cell similar to [4], [7].

The received signal at cell 1 in phase 0 and phase 1 are given by:

$$
\begin{aligned}
& \mathbf{Y}(0)=\mathbf{X H}_{1,1}+\sum_{l=2}^{L} \alpha_{l, 1} \mathbf{X H}_{l, 1}+\mathbf{Z}(0), \\
& \mathbf{Y}(1)=\sum_{l=2}^{L} \alpha_{l, 1} \mathbf{X H}_{l, 1}+\mathbf{Z}(1)
\end{aligned}
$$

where $\alpha_{l^{\prime}, l}$ is the parameter that stands for the asynchronous effect related to timing offset between the transmissions at cell $l^{\prime}$-th and at cell $l$-th [8] and it is assumed to be constant during $L+1$ phases; $\mathbf{Z}(0), \mathbf{Z}(1) \in \mathbb{C}^{M \times Q}$ is the noise matrix drawn at phase 0 and phase 1 , respectively.

At the $l$-th phase, the $l$-th cell stays silent, while the other $L-1$ cells are transmitting. The received signal at cell 1 in the $l$-th phase $2 \leq l \leq L$, is given by:

$$
\mathbf{Y}(l)=\mathbf{X H}_{1,1}+\sum_{l^{\prime}=2, l^{\prime} \neq l}^{L} \alpha_{l^{\prime}, 1} \mathbf{X H}_{l^{\prime}, 1}+\mathbf{Z}(l),
$$

where $\mathbf{Z}(l) \in \mathbb{C}^{M \times Q}$ is the noise matrix drawn at the $l$-th phase.

Based on the received signal from (4)-(7), we propose two channel estimators which can completely remove pilot contamination.

\section{A. Proposed Estimator 1}

The idea behind this proposed estimator is to promote the $\mathrm{BS}$ to run channel estimation as soon as it receives interference. 
It is realized that all inter-cell interference at cell 1 in (4) is contained in (5). Subtracting (4) by (5) we obtain:

$$
\widetilde{\mathbf{Y}}_{1}=\mathbf{Y}(0)-\mathbf{Y}(1)=\mathbf{X H}_{1,1}+\widetilde{\mathbf{Z}}_{1}
$$

where $\tilde{\mathbf{Z}}_{1}=\mathbf{Z}(0)-\mathbf{Z}(1)$. Because the entities of $\mathbf{Z}(0)$ and $\mathbf{Z}(1)$ are mutually independent, it is easy to show that the entities of $\tilde{\mathbf{Z}}_{1}$ are also i.i.d. complex Gaussian random variables with zero mean and variance $\sigma_{\tilde{z}_{1}}^{2}=2 \sigma^{2}$.

Applying the LS estimation to (7) we obtain:

$$
\widehat{\mathbf{H}}_{C E-1}=\left(\mathbf{X}^{H} \mathbf{X}\right)^{-1} \mathbf{X}^{H} \widetilde{\mathbf{Y}}_{1}=\mathbf{H}_{1,1}+\frac{1}{K} \mathbf{X}^{H} \widetilde{\mathbf{Z}}_{1} .
$$

It is observed from (8) that the pilot contamination is completely eliminated.

\section{B. Proposed Estimator 2}

The proposed estimator 2 aims to exploit all $L+1$ channel observations in order to improve the channel estimation accuracy. During $L-1$ phases $2 \leq l \leq L, \mathbf{Y}(l)$ does not contain interference from users in the $l$-th cell. If we take the sum of all $\mathbf{Y}(l)$ for $2 \leq l \leq L$, it contains $L-1$ times the pilot signal of interest $\mathbf{X H}_{1,1}$ and $L-2$ times the sum of all intercell interference $\sum_{l=2}^{L} \mathbf{X H}_{l, 1}$. Based on this observation, the proposed estimator 2 is given by:

$$
\begin{aligned}
\widetilde{\mathbf{Y}}_{2} & =\mathbf{Y}(0)+\sum_{l=2}^{L} \mathbf{Y}(l)-(L-1) \mathbf{Y}(1) \\
& =\widetilde{\mathbf{X}} \mathbf{H}_{1,1}+\widetilde{\mathbf{Z}}_{2},
\end{aligned}
$$

where $\widetilde{\mathbf{X}}=L \mathbf{X}$ and $\widetilde{\mathbf{Z}}_{2}=\mathbf{Z}(0)+\sum_{l=2}^{L} \mathbf{Z}(l)-(L-1) \mathbf{Z}(1)$. Again, since the entities of $\mathbf{Z}(l), 0 \leq l \leq L$, are mutually independents, the elements of $\widetilde{\mathbf{Z}}$ are proved to be i.i.d. complex Gaussian random variables with zero mean and variance $\sigma_{\widetilde{z}_{2}}^{2}=$ $\left(L^{2}-L+1\right) \sigma^{2}$. In addition, we have $\widetilde{\mathbf{X}}^{H} \widetilde{\mathbf{X}}=L^{2} K \mathbf{I}_{M}$.

Applying the LS estimation to (9) with respect to pilot signal $\widetilde{\mathbf{X}}$ we obtain:

$$
\begin{aligned}
\widehat{\mathbf{H}}_{C E-2} & =\left(\widetilde{\mathbf{X}}^{H} \widetilde{\mathbf{X}}\right)^{-1} \widetilde{\mathbf{X}}^{H} \widetilde{\mathbf{Y}}_{2} \\
& =\mathbf{H}_{1,1}+\frac{1}{L^{2} K} \widetilde{\mathbf{X}}^{H} \widetilde{\mathbf{Z}}_{2} .
\end{aligned}
$$

Clearly, pilot contamination is completely cancelled out in estimator 2. It is easily realized that estimator 2 yields better performance than estimator 1 because it exploits more observations. In order to apply estimator 2 , all cells have to wait for $L+1$ phases. Whereas in estimator 1 , the $l$-th cell, $l \leq L$, only needs to wait for $l+1$ phases to start estimating. We also note that MMSE estimator can be used in (7) and (9). However, we have observed in our scheme that the improvement of MMSE estimator over LS estimator is negligible.

\section{Channel Estimation Error Analysis}

In this subsection, we analyze the performance of the proposed estimators in term of average MSE, which is computed as follows:

$$
\bar{\Xi}_{i}=\mathbb{E}\left\{\frac{1}{M Q} \sum_{m=1}^{M} \sum_{q=1}^{Q}\left\|\boldsymbol{\Xi}_{i}\right\|_{F}^{2}\right\}, i=1,2,
$$

where $\boldsymbol{\Xi}_{i}=\widehat{\mathbf{H}}_{C E-i}-\mathbf{H}_{1,1}$ is the estimation error of the estimator $i$ and $\|.\|_{F}$ denotes the Frobenius norm.

Theorem 1: The average MSE of the proposed estimators is given by:

$$
\bar{\Xi}_{1}=\frac{2 \sigma^{2}}{K}, \text { and } \bar{\Xi}_{2}=\frac{\left(L^{2}-L+1\right) \sigma^{2}}{L^{2} K} .
$$

Proof: From (8) and (10) we obtain:

$$
\boldsymbol{\Xi}_{1}=\frac{1}{K} \mathbf{X}^{H} \widetilde{\mathbf{Z}}_{1} \text {, and } \boldsymbol{\Xi}_{2}=\frac{1}{L^{2} K} \widetilde{\mathbf{X}}^{H} \widetilde{\mathbf{Z}}_{2} .
$$

Let $\Xi_{i}[m, q]$ be the entity of $\boldsymbol{\Xi}_{i}$ at row $m$-th and column $q$-th, and $\mathbf{x}_{j}$ be the $j$-th column of the matrix $\mathbf{X}$. Then we have:

$$
\begin{aligned}
& \Xi_{1}[m, q]=\frac{1}{K} \mathbf{x}_{[m]}^{H} \widetilde{\mathbf{z}}_{1[q]}, \\
& \Xi_{2}[m, q]=\frac{1}{L^{2} K} \widetilde{\mathbf{x}}_{[m]}^{H} \widetilde{\mathbf{z}}_{2[q]} .
\end{aligned}
$$

Because the entities of $\widetilde{\mathbf{Z}}_{1}$ are i.i.d. Gaussian random variables, with zero mean, $\Xi_{i}[m, q]$ is also i.i.d. Gaussian variable with zero mean. Let $x_{[m]}[k]$ be the $k$-th element of $\mathbf{x}_{[m]}$, then the variance of $\Xi_{1}[m, q]$ can be computed as follows:

$$
\begin{aligned}
\mathbb{E}\left\{\left|\Xi_{1}[m, q]\right|^{2}\right\} & =\frac{1}{K^{2}} \sum_{k=1}^{K}\left|x_{[m]}[k]\right|^{2} \sigma_{\widetilde{z}_{1}}^{2} \\
& =\frac{\sigma_{\widetilde{z}_{1}}^{2}}{K}=\frac{2 \sigma^{2}}{K} .
\end{aligned}
$$

Similarly, taking into account that $\sum_{k=1}^{K}\left|\widetilde{x}_{[m]}[k]\right|^{2}=L^{2} K$, we obtain:

$$
\mathbb{E}\left\{\left|\Xi_{2}[m, q]\right|^{2}\right\}=\frac{\sigma_{\widetilde{z}_{2}}^{2}}{L^{2} K}=\frac{\left(L^{2}-L+1\right) \sigma^{2}}{L^{2} K} .
$$

Substituting (15) and (16) into (11) will complete the prove of Theorem. 1 .

From Theorem 1, we can see that the estimator 2 significantly reduces the average MSE compared to estimator 1 (compare $\bar{\Xi}_{1}$ with $\bar{\Xi}_{2}$ ). The average MSE of estimator 1 does not rely on the network size and both estimators are independent from the channel fading model.

\section{Numerical Results}

We demonstrate the performance of our proposed estimators in hexagonal cellular networks. The users are assumed to be uniformly distributed in each cell. Otherwise stated, the basic simulation parameters are as follows: number of cells $L=7$ with cell radius equal $1000 \mathrm{~m}$, number of antennas $Q=20$, number of pilots $K=8$ and the pathloss exponent is equal to 3 .

Normalized MSE (NMSE) of the proposed estimators are compared with that of referenced schemes including: i) Aligned pilots with covariance-aided estimator [4], ii) Time-shifted pilots [6], and iii) the proposed scheme in [7] (named [7]). 


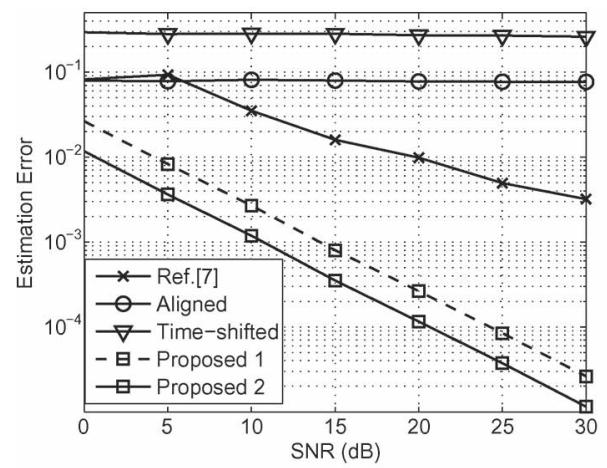

(a)

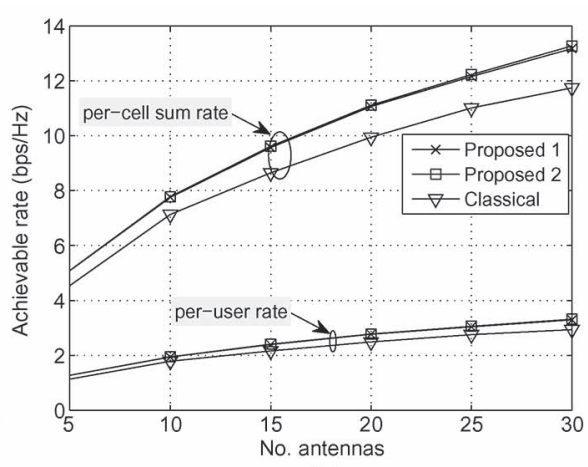

(b)

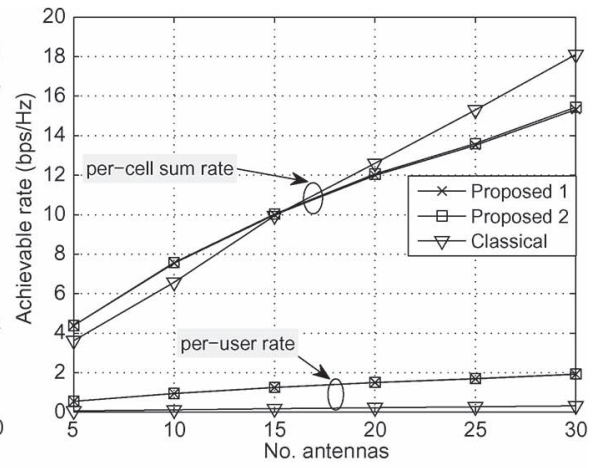

(c)

Fig. 1. (a)-Estimation errors comparison among estimators and (b) and (c)-Sum rate and per-user rate comparison between the proposed scheme and the classical scheme. SNR $=20 \mathrm{~dB}$ at cell edge. (b): $M=4$ users in all schemes and (c): 8 served users in the proposed scheme, 56 served users in the classical scheme.

We note that [4] needs to know the second-order statistics of all user channels. Although [4] proposed a coordinated pilot assignment scheme, it only allows one user to be activated at a time, and therefore is not applicable in this scenario. The NMSE is defined for estimator $i$ as follows:

$$
\mathrm{NMSE}^{i} \triangleq \frac{\sum_{m=1}^{M} \sum_{q=1}^{Q}\left|\Xi_{i}[m, q]\right|^{2}}{\sum_{m=1}^{M} \sum_{q=1}^{Q}\left|H_{1,1}[m, q]\right|^{2}}, i=1,2 .
$$

For fair comparison, we fix the channel estimation period equal to 56 symbols. Consequently, the proposed estimator and [7] use $K=8$ pilot symbols, while the others employ $K L=56$ pilot symbols. It is shown in Fig. 1(a) that the proposed estimators significantly outperform other estimators. By taking into consideration all interference, the estimator 2 further improves the NMSE about $4 \mathrm{~dB}$ over the estimator 1 . We note that the performance of the aligned and time-shifted estimators does not improve as the SNR increases because the former is limited by pilot contamination while the latter is bounded by strong interfered downlink signal [6].

We also compare the downlink per-cell sum rate and per-user achievable rate of the proposed scheme with those of the classical aligned-pilot scheme [4] (Fig. 1(b) and (c)). In both cases, the proposed scheme achieves much higher per-user achievable rate. For the same number of served users (Fig. 1(b)), our scheme also supports much higher (per-cell) sum rate than the classical scheme. It is noted that in this case long pilot sequence might be used in the classical scheme if the pilots in all users are mutually independent. However, this will require centralized pilot assignment for the whole network which is challenging and costs too much overhead signal. Considers the full-load case where the number of users are equal to the number of the pilot symbols ${ }^{1}$ (Fig. 1(c)), our scheme achieves higher percell sum rate than the classical scheme when the number of BS antennas is small and medium because more users in the classical scheme results in stronger inter-cell interference. At high antenna number, the classical scheme achieves higher percell sum rate. In conclusion, the proposed scheme is more effective if the number of active users is not too large.

\section{CONCLUSiON}

We have proposed two channel estimation schemes for multiantenna multicell cellular networks that completely eliminate pilot contamination. Exact closed-form expression for average MSE is provided. More importantly, the proposed estimators does not relay on the assumption that each BS knows the second-order statistics of desired and/or interfering user channels.

\section{REFERENCES}

[1] J. Jose, A. Ashikhmin, T. L. Marzetta, and S. Vishwanath, "Pilot contamination problem in multi-cell tdd systems," in Proc. IEEE ISIT, 2009, pp. 2184-2188.

[2] J. Hoydis, S. T. Brink, and M. Debbah, "Massive mimo: How many antennas do we need?" in Proc. 49th Annu. Allerton Conf. Commun., Control, Comput., 2011, pp. 545-550.

[3] T. L. Marzetta, "Noncooperative cellular wireless with unlimited numbers of base station antennas," IEEE Trans. Wireless Commun., vol. 9, no. 11, pp. 3590-3600, Nov. 2010.

[4] Y. Haifan, D. Gesbert, M. Filippou, and L. Yingzhuang, "A coordinated approach to channel estimation in large-scale multiple-antenna systems," IEEE J. Sel. Areas Commun., vol. 31, no. 2, pp. 264-273, Feb. 2013.

[5] K. Appaiah, A. Ashikhmin, and T. L. Marzetta, "Pilot contamination reduction in multi-user tdd systems," in Proc. IEEE ICC, 2010, pp. 1-5.

[6] F. Fernandes, A. Ashikhmin, and T. L. Marzetta, "Inter-cell interference in noncooperative TDD large scale antenna systems," IEEE J. Sel. Areas Commun., vol. 31, no. 2, pp. 192-201, Feb. 2013.

[7] J. Zhang et al., "Pilot contamination elimination for large-scale multipleantenna aided OFDM systems," IEEE J. Sel. Topics Signal Process., vol. 8, no. 5, pp. 759-772, Oct. 2014.

[8] Y. Medjahdi, M. Terre, D. Le Ruyet, D. Roviras, and A. Dziri, "Performance analysis in the downlink of asynchronous OFDM/FBMC based multi-cellular networks," IEEE Trans. Wireless Commun., vol. 10, no. 8, pp. 2630-2639, Aug. 2011. 